\title{
V12 Host soluble factors induced by moreau BCG, in vitro, in the initiation of monocyte apoptosis in healthy donors
}

Leandro Peres' ${ }^{1}$, Suelen Marinho¹, Jéssica Lima1', Mariana Siqueira1, Thaize Pedro', Dolores Silva1', Carlos Ponte' ${ }^{1}$, Paula de Luca ${ }^{2}$, Luiz Castello Branco ${ }^{1}$, Paulo Antas ${ }^{1}$

${ }^{1}$ Laboratório de Imunologia Clínica, Oswaldo Cruz Institute, Fiocruz

2 Laboratório de Imunoparasitologia, Oswaldo Cruz Institute, Fiocruz

Introduction: Tuberculosis (TB) remains the world's leading cause of mortality.For its control, studies of TB vaccines are needed. Since BCG is the only vaccine against TB currently in use, studies addressing the protective role of BCG are urgent required.

Objective: To evaluate the mechanisms by which BCG develops its protective action.

Methodology: Cohorts of HIV-negative voluntaries have been enrolled: Adult Control Donors (CD; $n=18)$ and neonates Umbilical Vein (UV; $n=10$ ). BCG Moreau was primarily used for in vitro monocyte infection at both $24 \mathrm{~h}$ and $48 \mathrm{~h}$. After that, harvested conditioned medium (CM) was added to autologous resting cells for an additional $24 \mathrm{~h}$ or 120h, and Annexin V-FITC and PI were used for apoptosis detection. Also, in those cultures the remaining CD4+ lymphocytes were stained for PD-1 and CD25/FoxP3, and concurrent caspases were detected in monocytes. Supernates were assayed for Nitric Oxide (NO2).

Results: P levels were setup at $<0.05$. Here, conditioned medium (CM) induced higher apoptosis levels in the Adult Control Donors (CD) group only. NO2 were released equally during BCG infection in both groups, but higher levels were found in CD, when compared to UV group $(p<0.05)$. For PD-1, higher levels were also observed in CD-derived CD4 lymphocytes only. On the other hand, mean levels of Caspases in monocytes and Treg cells (CD4+/CD25high+/FoxP3+) were not induced in either group of individuals, but expression of CD25dim+ as an activation marker was dependent on BCG infection only in the UV group.

Conclusion: The vast majority of the world's current population has been vaccinated with $B C G$, with the possible requirement for a booster immunization in adulthood for TB protection. Sustained data have shown an enhancement of in vitro apoptosis levels in adult monocytes during long-term incubation with CM from BCG Moreau RDJ cultures, but neonate monocytes remained viable when cultured with autologous $\mathrm{CM}$. That was followed by higher NO2 released and PD-1 expression on CD4+ T cells from the primed group, but not in naïve individuals, although only neonates showed in vitro activated lymphocytes. A 
booster immunization with BCG may protect the immunized individuals and further studies are needed to better evaluate these findings.

Keywords: Tuberculosis, BCG, Apoptosis 